\title{
New perspectives and recommendations for anticoagulant therapy post orthopedic surgery
}

\author{
Marcelo Kropf $^{1 *}$, Cleidson Alves Bergami ${ }^{1}$, Felipe Dias Leal ${ }^{1}$, Claudia Oliveira Dias Passos ${ }^{1}$, Zilda \\ de Santana Gonsalves', Isabela Laudares Marques², Isabela Azevedo Mota², Marcele Lima Monte \\ Gonçalves $^{2}$
}

\author{
'Pharmacy Unit. National Institute of Traumatology and Orthopedics, Instituto Nacional de Traumatologia e Ortopedia, \\ ${ }^{2}$ Residency Course: Specialist in Hospital Pharmacy, Fluminense Federal University
}

\begin{abstract}
Anticoagulant therapy is essential for the prevention of risks associated with the formation of thrombus in patients after surgery, especially in orthopedics. Recently, new oral anticoagulants were introduced in the therapeutic arsenal. This fact is important, because the current drug of choice in clinical practice is enoxaparin, a low molecular weight heparin. As all injecting drugs, enoxaparin may reduce patients' adherence to treatment by dissatisfaction with and resistance to the administration. This article reviews the available literature on the overall utility of these innovative medicines, approaching the pharmacology, the compared efficacy in relation to current agents, and the potential targets for new agents, as well as points to new trends in research and development. The article also contributes with a practical guide for use and recommendations to health professionals, especially focusing on the reversibility of hemorrhagic events, and discusses the importance of convenience/satisfaction of use, the cost of treatment, and the risk-benefit profile for patients.
\end{abstract}

Uniterms: Oral anticoagulants/use/patient compliance. Injectable anticoagulants/use/patient compliance. Anticoagulant therapy. Enoxaparin/use. Dabigatrana/use. Rivaroxabana/use. HPBM drugs/use.

A terapia anticoagulante é fundamental para a prevenção de riscos associados à formação de trombos em pacientes pós-cirúrgicos, principalmente em ortopedia. Recentemente, novos anticoagulantes orais foram introduzidos no arsenal terapêutico. Tal fato é importantíssimo, visto que o atual medicamento de primeira escolha na prática clínica é a enoxaparina, uma heparina de baixo peso molecular. Por ser de uso injetável, a enoxaparina pode diminuir a adesão do paciente ao tratamento, devido à insatisfação e à resistência quanto à via de administração. Este artigo revisa a literatura disponível sobre a utilidade total desses medicamentos inovadores ao abordar a farmacologia, a eficácia em comparação com os agentes atuais e os alvos potenciais para novos agentes, bem como aponta as novas tendências em pesquisa e desenvolvimento. $\mathrm{O}$ artigo também contribui com um guia prático de uso e recomendações aos profissionais de saúde, com um enfoque especial sobre a reversibilidade de eventos hemorrágicos e, finalmente, discute a importância da conveniência/satisfação de uso, o custo de tratamento e o perfil risco-benefício para o paciente.

Unitermos: Anticoagulantes orais/use/adesão ao tratamento. Anticoagulantes injetáveis/uso/adesão ao tratamento. Terapia anticoagulante. Enoxaparina/uso. Dabigatrana/uso. Rivaroxabana/uso. Fármacos $\mathrm{HPBM}$ /uso.

\section{INTRODUCTION}

All drug therapy needs to satisfy several requirements for good quality in health care. Safety and efficacy

*Correspondence: M. Kropf. Unidade de Farmácia, Instituto Nacional de Traumatologia e Ortopedia - INTO. Avenida Brasil, 500 - São Cristóvão 20940-070 - Rio de Janeiro - RJ, Brasil. E-mail: mkropf@into.saude.gov.br of drugs are extremely important, but not unique, properties to consider. Nowadays, a convenient treatment increases adherence, and directly contributes to drug therapy success. In addition to these three requirements, the cost of treatment is also a constant concern, because a safe, effective and convenient therapy for the population is extremely limited, due to the high costs related (Wittkowsky, 2010). 
Thesearch for anticoagulants with acceptable efficacy and safety associated with patient compliance has been extensive, and several drugs were developed. A better understanding about the coagulation cascade allowed the development of drugs with action mechanisms different of those already existent, which directly act on previously unexplored coagulation factors. The evolution of recombinant DNA technology, in addition to the production of biopharmaceuticals on a large scale, promoted the expansion inthe number of anticoagulant drug prototypes, based on substances found in biological systems (Walsh, 2007).

Despite the extensive use of heparin and low molecular weight heparins (LMWHs) in thromboprophylaxis after orthopedic surgeries, the subcutaneous administration also decreases patient adherence to treatment, which is viewed with concern by health professionals involved in drug therapy. Therefore, the development of oral anticoagulants which are able to replace heparin and LMWHs, with improved safety and efficacy compared to warfarin, is a matter of great expectation.

This review article aims to inform health professionals about the development of anticoagulant drugs and their use in patients who underwent orthopedic surgeries. Additionally, this paper comprises the relevance of new oral anticoagulants) as dabigatran and rivaroxaban, formulating a practical guide for use, as well as new trends in research and development.

\section{Pharmacology and potential targets for new agents}

Hemostasis, a crucial event to life, is defined as the interruption of blood loss by injured vessels. Blood must remain fluid within the vasculature, and still quickly coagulate when exposed to non-endothelial surfaces, such as regions of vascular injuries. When intravascular thrombi are formed, there is an activation of the fibrinolysis system in order to restore the normal blood flow. Under normal conditions, a delicate balance is established with the purpose of avoiding thrombosis and bleeding. This balance is obtained by physiological fibrinolysis, without excessive pathological fibrigenolysis. In the first step of thrombus formation, platelets adhere to macromolecules in the subendothelial regions of the injured blood vessel, and then aggregate to form a primary hemostatic cover. Then, platelets stimulate local activation of plasma coagulation factors, resulting at the end in a fibrin clot. Later, as the injury is healed, both the platelet aggregate and the fibrin clot are destroyed (Majerus, Tollefsen, 2006).

The conversion of fibrinogen, a floating soluble peptide, into insoluble threads of fibrin is catalysed by throm- bin (factor IIa), and it is the main event of the coagulation cascade. Moreover, thrombin activates factor XIII as well, which crosslinks the fibrin fragments and consequently stabilizes the clot. Fibrin formation involves a complex cascade of reactions, whose components (factors) are present in blood as inactive precursors, but can be activated by enzymatic proteolysis. The activation of a small amount of one factor catalyses the activation of larger quantities of the following factor. That cascade provides a sophisticated mechanism of signal amplification (Rang et al., 2003). However, under normal conditions, such amplification is carefully controlled by physiological inhibitors such as inhibitor of tissue factor pathway, antithrombin III (ATIII), an inhibitor of thrombin, and activated protein Can inhibitor of factors VIIIa and Va (Breitenstein, Tanner, Lüscher, 2010). There are two pathways which culminate in fibrin formation: 1) the intrinsic pathway - all its components are present in the blood; and 2) extrinsic pathway-some factors are external to the vasculature. Both finally activate the common pathway (Figure 1).

Understanding of the coagulation cascade allowed the development of anticoagulant drugs, or, in other words, substances used to prevent unwanted blood clotting, mainly when endogenous anticoagulant mechanisms are defective or insufficient. Some severe pathological conditions such as venous thromboembolism (VTE), deep vein thrombosis (DVT), and pulmonary embolism (PE) require the therapeutic use, in the short or long term, of this class of drugs. Anticoagulant therapy is also recommended for the long-term prevention of ischemic stroke in patients with atrial fibrillation and for the prevention of recurrent myocardial infarction in patients with acute coronary syndromes (ACSs), such as unstable angina or non-ST elevated myocardial infarction (Turpie, 2007).

Since the discovery of heparin, in 1916, the development and use of these drugs in the prevention and treatment of thromboembolic events have been growing worldwide, especially with population aging. For instance, patients who are undergoing some surgeries such as total hip arthroplasty (THA) and total knee arthroplasty (TKA) have high risk of developing VTE and proximal and total DVT. This threat has decreased significantly in the last 20 years, with the introduction of thromboprophylaxis, which is currently performed using oral or parenteral anticoagulant drugs (Weitz, Hirsh, Samama, 2008).

Nowadays, the most common available anticoagulant drugs include the heparinoids, unfractionated heparin (UFH), and low molecular weight heparins (LMWHs) such as enoxaparin, dalteparin and tinzaparin, which emerged due to their greater safety, efficacy and convenience of administration when compared to UFH. Other 


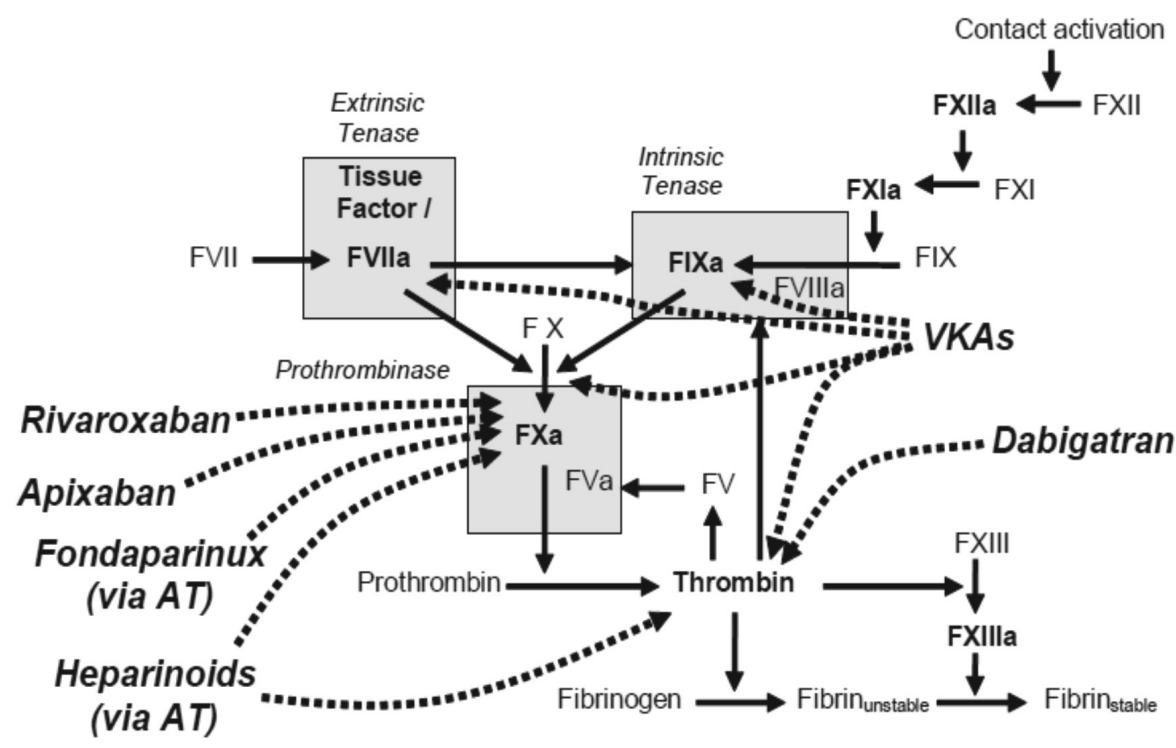

FIGURE 1 - Classic anticoagulant drugs and their targets in the coagulation cascade (Lassen, Laux, 2008).

important drugs are the vitamin $\mathrm{K}$ antagonists (VKA), such as warfarin, and indirect inhibitors (because they require the action of ATIII) of FXa, such as fondaparinux and idraparinux (Figure 1).

However, these therapies have some important limitations, such as the risk of thrombocytopenia, the parenteral use of heparins and fondaparinux, the monitoring need, low therapeutic indexes, and drug interactions with warfarin, which underlined the need to develop new agents. Due to this situation, new oral drugs were recently produced. As examples of this type of drugs, it can be pointed out the direct thrombin inhibitor (with independent action of ATIII) dabigatran, and Factor Xa inhibitorssuch as rivaroxaban and apixaban. Ximelagatran, another direct thrombin inhibitor, was removed from the European Pharmacopoeia, due to liver toxicity induced by this substance (Samama, Gerotziafas, 2010). Other molecules, even in studies, appear as candidates

ORAL

\section{PARENTERAL}

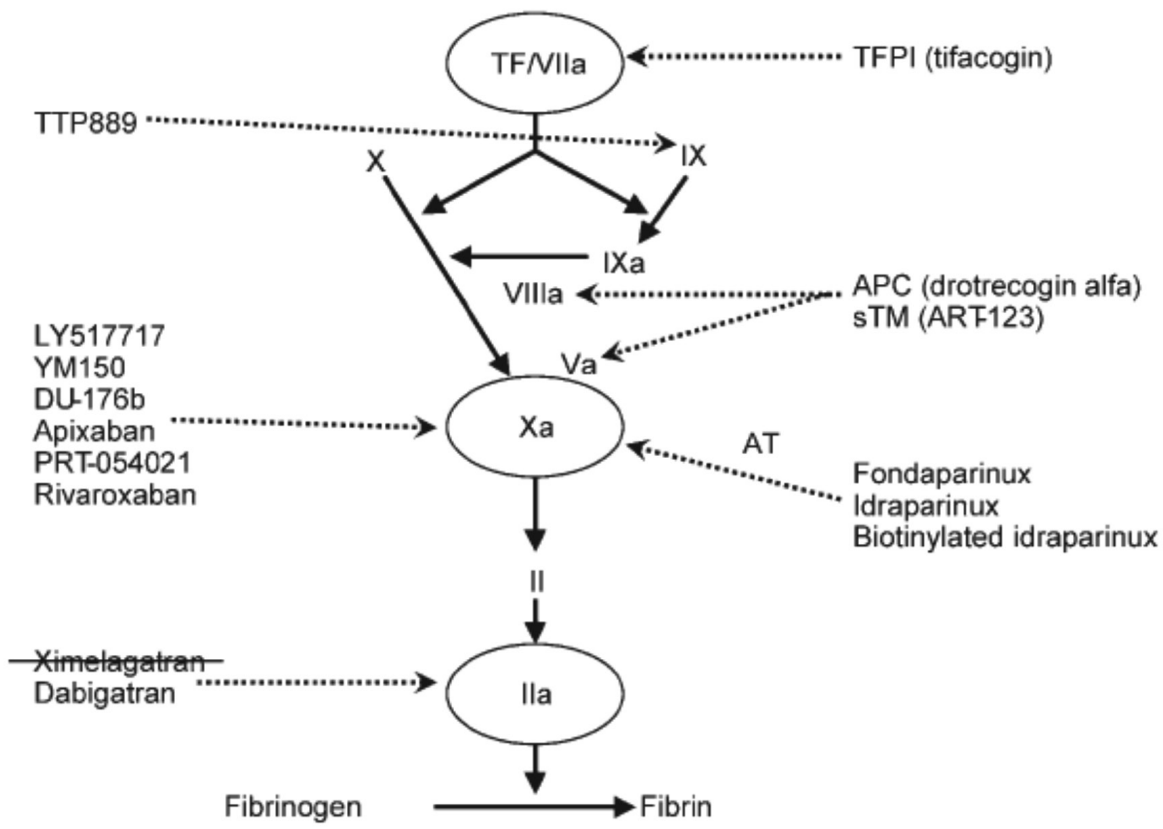

FIGURE 2 - Targets for new anticoagulant drugs in the coagulation cascade (Turpie, 2007). 
for anticoagulant therapies with different targets in the coagulation cascade (Figure 2).

There are important features about the new anticoagulants, which need to be discussed. One example is their mechanisms of action, involving the direct or indirect inhibition of factors Xa and IIa, target specificities, intensity of anticoagulation, type of target connection, and potential antigen actions. There is a disagreement among experts about the drugs that would bring a better risk-benefit ratio, because only a small number of trials were done.

One limitation to the clinical evaluation of new anticoagulants is the low sensitivity of current coagulation tests, which lead to an incorrect evaluation of the risk of bleeding related to each drug. For instance, fondaparinux and idraparinux hardly change the prothrombin time (PT) and the activated partial thromboplastin time (APTT), even when administered in doses so high that they trigger hemorrhage episodes. Prolonged PT induced by anticoagulant drugs as rivaroxaban cannot be expressed by the international normalized ratio (INR). It is applied only for the VKAs, because an INR enhanced by a new anticoagulant (inhibitor of only one factor of the coagulation cascade) can not be compared to an INR of a VKA (inhibitor of several factors of this cascade).

It is necessary to investigate the reasons that lead to fondaparinux/idraparinux and rivaroxaban be or not different in increasing PT. A possible reason is that rivaroxaban inhibits free Factor Xa and also Factor Xa linked to phospholipids within the prothrombinase complex, while fondaparinux and idraparinux only inhibit free Factor Xa.

Some other properties should to be investigated, as the possibility of cardiovascular complications related to the rebound effect of coagulation with the discontinuation of treatment and, especially, the existence of an antidote, preferably specific for drugs with long half-life. An important stage of clinical trials is to determine the therapeutic window, especially for drugs such as dabigatran, which displays a strong interdependence between the concentration of the drug and clinical response based on the number of occurrences of thromboembolic events (Samama, Gerotziafas, 2010).

\section{Direct inhibitors of Factor Xa for parenteral use}

Fondaparinux is a synthetic pentassaccharide similar to that of heparin, with half-life of approximately 17 hours, increasing to approximately 20 hours in elderly people. This long half-life allows a regimen of just one daily subcutaneous administration. In surgical patients, the therapeutic dose is $2.5 \mathrm{mg}$, while in patients treating severe
DVT or PE the therapeutic dose may reach $7.5 \mathrm{mg}$, which is considered safe for patients between 50 and $100 \mathrm{~kg}$. For patients less than $50 \mathrm{~kg}$ and over $100 \mathrm{~kg}$, daily doses of 5 $\mathrm{mg}$ and $10 \mathrm{mg}$ are respectively suggested.

Idraparinux is a synthetic pentassaccharide similar to fondaparinux; however, idraparinux have a greater anticoagulant activity, mainly due to its greater affinity for antithrombin and its long half-life (80 to 130 hours). These properties allow it to be administered subcutaneously, just once a week. Clinical trials have shown its efficacy and safety for treatment of DVT only, although its anticoagulant effect after discontinuation of treatment was observed. Studies about its use in the treatment of PE did not produce good results due to the recurrence of PE in the first five days of treatment.

DX-9065a is a nonpeptidic direct inhibitor of Factor $\mathrm{Xa}$ that is administered parentally. It has a dose-dependent half-life that ranges from 40 minutes to 5 hours, and is cleared by kidneys. This drug was evaluated in patients with non-ST-elevation ACS, and in patients undergoing percutaneous coronary intervention. In a trial on ACS, 402 patients were randomized to weight-adjusted heparin or to low- or high-dose DX-9065a. There were no significant decreases in deaths, myocardial infarction, need for urgent revascularization, or ischemia. Cases of serious bleeding occurred in both groups of patients, without statistically significant differences (Weitz, Hirsh, Samama, 2008).

Otamixaban, a non-competitive inhibitor of FXa, is administered intravenously and has a half-life of 2-3 hours. It is excreted unchanged in urine, whereas metabolites appear in the feces. A phase II study compared a 24-hour otamixaban infusion with placebo in patients with stable coronary artery disease. The addition of otamixaban to usual medications did not cause bleeding, and otamixaban produced a rapid and sustained increase in anti-factor Xa activity. A phase II trial comparing otamixaban with heparin in patients undergoing nonurgent percutaneous coronary interventions demonstrated greater reductions in the levels of prothrombin fragment with otamixaban than with heparin; no difference in the rate of major bleeding was observed.

\section{Direct inhibitors of Factor Xa for oral use}

The advantages of oral anticoagulants compared to parenteral are obvious, mainly considering the time of treatment, which sometimes lasts weeks, months, and even years. Among the new oral anticoagulants, there are rivaroxaban, apixaban and edoxaban, all of which have molecular mass of approximately $500 \mathrm{Da}$. These compounds potentially and selectively inhibit Factor Xa and, in increasing doses, increase PT more than PTTA. 
Rivaroxaban is quickly absorbed, reaches its maximum blood concentration 2 to 4 hours after administration, has oral bioavailability of $80 \%$, and is cleared mainly by kidneys. In Europe, this drug is indicated for primary prevention of thrombotic events subsequent to THA and TKA surgeries in adults. Its use in preventing stroke in patients with non-valvular atrial fibrillation is still under investigation. Clinical studies comparing the efficacy of rivaroxaban and enoxaparin in reducing cases of VTE after THA and TKA surgeries showed a significant reduction of $50 \%$ in the cases of VTE between groups treated with rivaroxaban when compared with enoxaparin-treated groups. There was no significant difference between groups when cases of bleeding were compared. Moreover, there is still a discussion about the risk of hemorrhage associated with treatment with rivaroxaban, because studies conducted by two different research groups reached opposite conclusions. The rebound phenomenon after discontinuation of cardiovascular treatment with rivaroxaban should be further investigated, because there is some degree of concern expressed in literature.

\section{Direct inhibitors of thrombin}

Direct inhibitors of thrombin are different from heparins, whose anticoagulant activities are mediated by antithrombin. Some examples of this type of drugs are hirudin, its derivative bivalirudin, argatoban, ximelagatran, and dabigatran, which is discussed below.

Dabigatran is orally administered as a prodrug - dabigatran etexilate - and was initially studied for prophylaxis after THA and THK surgeries, and then for the treatment of acute episodes of VTE. Its use in the prevention of VTE cases and stroke in patients with non-valvular atrial fibrillation is still also under clinical investigation. A clinical study showed that dabigatran was not as efficient as enoxaparin in preventing VTE, due to high rates of distal DVT, even with equivalent rates of VTE. About DVT and $\mathrm{PE}$ rates and mortality due to VTE, there was no significant difference among the drugs analyzed. Even with no reports of hepatic toxicity, it isnot recommended to administer dabigatran in patients with severe liver failure or elevated liver enzymes or bilirubin. The risk-benefit ratio in elderly patients predisposed to cardiovascular events or with renal failure was considered satisfactory (Weitz, Hirsh, Samama, 2008).

\section{Inhibitors of Factor Va}

Factor Va is the main target of activated protein C, which acts as an anticoagulant substance by proteolytic degradation and inactivation of factor Va, a key cofactor in thrombin generation.

ART-123, a recombinant analogue of the extracellular domain of thrombomodulin, binds thrombin and enhances its capacity to activate protein $\mathrm{C}$. This drug has nearly $100 \%$ bioavailability after subcutaneous administration, and a half-life of 2 to 3 days. Plans for further development of this agent are uncertain ${ }^{8}$.

Drotrecogin, a recombinant form of activated protein $C$, is approved for treatment of patients with severe sepsis. Approval for this indication was based on a trial comparing drotrecogin with placebo in 1,690 patients with severe sepsis. In this study, drotrecogin significantly reduced the mortality of patients in 28 days. However, other two clinical trials were stopped prematurely, due to lack of efficacy and the potential to cause harm because of bleeding ${ }^{8}$.

\section{Inhibitors of Factor VIla}

Drugs that target the factor VIIa inhibit the initiation of coagulation. Only parenteral agents in this category have reached phase II or III clinical testing. These include tifacogin, recombinant nematode anticoagulant peptide (NAPc2) and inactive factor VIIa (factor VIIai).

Ticofagin, a recombinant form of tissue factory pathway inhibitor expressed in Saccharomyces cerevisae, has been evaluated in patients with sepsis. This drug has a half-life of minutes, necessitates intravenous infusion, and is cleared by liver. In a phase II trial, 44210 sepsis patients were randomized to receive one or two doses of tifacogin by continuous infusion or placebo for 4 days, resulting in $20 \%$ relative reduction in 28-day mortality. Differences about major bleeding were not statistically significant. Based on these results, a phase III trial that compared tifacogin with placebo in severe sepsis patients was performed. The primary end point, 28-day mortality, was similar with tifacogin and placebo, while the rate of bleeding was significantly higher with tifacogin (Weitz, Hirsh, Samama, 2008).

Recombinant NAPc2, an 85-amino acid polypeptide originally isolated from the canine hookworm, Ancylostoma caninum, is expressed in yeast. This substance binds to a noncatalytic site on factor X or factor Xa. Once bound to factor Xa, the NAPc2/factor Xa complex inhibits tissue factor-bound factor VIIa. Because it binds factor X with high affinity, NAPc2 has a half-life of approximately 50 hours after subcutaneous injection. Consequently, the drug can be administered on alternate days.

Initial clinical studies focusing on thromboprophylaxis showed this drug has the same efficacy and safety 
when compared with LMWHs. However, prospective randomized studies are needed to confirm these findings. After these promising results, further studies focusing on the application of this drug on arterial thrombosis and as a substitute for heparin in patients undergoing PCI are being conducted.

In turn, recombinant factor VIIa, which has its active site irreversibly blocked, competes with factor VIIa for tissue factor binding, thereby attenuating the initiation of coagulation by the factor VIIa/tissue factor complex. Based on promising results in animal models of thrombosis, factor VIIai, with or without adjunctive heparin, was compared with heparin alone in patients undergoing elective PCI. Factor VIIai, with or without adjunctive heparin, produced no significant reduction in the primary end point, a composite of death, myocardial infarction, need for urgent revascularization, abrupt vessel closure, use of Glycoprotein IIb/IIIa antagonists or heparin at day 7 or at hospital discharge. Rates of major bleeding were similar with factor VIIai and heparin. Because of these disappointing results, factor VIIai has not been developed further for treatment of arterial thrombosis (Weitz, Hirsh, Samama, 2008).

\section{Inhibitors of Factor IXa}

Both parenteral and oral factor IXa inhibitors are under development. The parenteral factor IXa inhibitor is an RNA aptamer (designated RB006) that binds factor IXa with high affinity. In phase I studies, this aptamer produced rapid anticoagulation as evidenced by a dose-dependent prolongation of the APTT. A positive aspect of RB006 is its potential for rapid neutralization by a complementary oligonucleotide (designated RB007). This drug-antidote pair is being developed for use in cardiopulmonary bypass surgery, or other indications where rapid anticoagulant reversal may be beneficial. An orally active direct factor IXa inhibitor also has been developed. Designated TTP889, this agent completed a phase IIa clinical trial that was negative (Weitz, Hirsh, Samama, 2008).

\section{Practical Guide for Use of Dabigatran and Rivaroxaban}

The search for an ideal anticoagulant aims at finding an oral agentthat, unlike warfarin, does not require a routine coagulation monitoring. Moreover, this ideal drug must have a wide therapeutic index, with low variability within and among patients; shall not have any interactions with other drugs or foods; shall have the ability of use in monotherapy, with no overlapping of parenteral anticoagu- lant drug, for use in acute and chronic thromboembolism, in hospital or at home, with an adequate safety profile; shall have a minimum risk of adverse effects; and, finally, its anticoagulant effect shall be easily reversible, with or without an antidote.

\section{Assays}

Among the new oral anticoagulants, dabigatran and rivaroxaban will be the objects of study of this practical guide. Indeed, a theoretic discussion about the superiority of use of factor Xa rivaroxaban versus factor IIa dabigatran as a target for anticoagulant therapy was always present in related research. For phase III clinical trials of dabigatran for TKA and THA, comparative studies with enoxaparin - RE-MOBILIZE (TKA, $n=2,615$ ), RE-MODEL (TKA, $\mathrm{n}=2,101)$, and RE-NOVATE (THA, $\mathrm{n}=3,494)-$ had very distinct demonstrative analyses. In RE-MOBILIZE (TKA, $n=2,615$ ), dabigatran was inferior to enoxaparin; in the other studies, equivalence between these drugs was observed. However, from a meta-analysis of available assays, the immediate conclusion is that there was no difference between dabigatran and enoxaparin in any of the endpoints analyzed. As for rivaroxaban, phase III clinical trials comparing it to enoxaparin - RECORD 1 (THA, $\mathrm{n}=$ 4,541), RECORD 2 (THA, $\mathrm{n}=2,509$ ), RECORD 3 (TKA, $\mathrm{n}=2,531)$ and RECORD 4 (TKA, $\mathrm{n}=3,148)$ - showed superiority of rivaroxaban to enoxaparin, and a meta-analysis confirmed these results (Wittkowsky, 2010).

\section{Pharmacokinetic parameters}

Pharmacokinetic parameters provide valuable information about these oral anticoagulants, as shown in Table I. Extensive discussions about these parameters are not the purpose of this paper. Instead, by providing a quick source of information, a discussion about the relevance of this review is intended.

Dabigatran exetilate, the prodrug of dabigatran, requires an acid environment for absorption. Each drug capsule contains multiple pellets that have a tartaric acid core that creates an acidic microenvironment to improve dissolution and absorption of dabigatran exetilate, independent of gastric $\mathrm{pH}$. Antacids and $\mathrm{H}_{2}$ blockers have no effect on this system or on the absorption of dabigatran etexilate. Proton pump inhibitors reduce the average area under the serum concentration versus time curve (AUC) of dabigatran by $22 \%$ and the average maximum serum concentration (Cmax) by 33\%, but these changes are not considered clinically relevant. Food simply delays the time to Cmax, and thus dabigatran exetilate can be taken orally 
with or without meals. In comparison, food not only delays the time to Cmax for rivaroxaban, it also increases the AUC by $28 \%$ and the Cmax by $41 \%$. In addition, administration with food appears to reduce interpatient variability of AUC and Cmax, thus increasing the predictability of plasma drug concentrations. Thus, it is recommended that rivaroxaban be taken with meals or within 2 hours after meals. Antacids and $\mathrm{H}_{2}$ blockers have no impact on rivaroxaban, but no information is currently available about the effect of proton pump inhibitors. The effect of food, antacids, $\mathrm{H}_{2}$ blockers and proton pump inhibitors on apixaban has not yet been reported (Wittkowsky, 2010).

About renal function, rivaroxaban shall be used with caution in patients with creatinine clearance (Clcr) of less than $30 \mathrm{~mL} / \mathrm{min}$, and is not recommended in patients with Clcr of less than $15 \mathrm{~mL} / \mathrm{min}$. There is much more to be learned about the safety of these agents in patients with mild moderate and severe renal impairment, as well as in those with changing renal function (Wittkowsky, 2010).

On the other hand, dabigatran appears to have less drug interaction potential than rivaroxaban, because its metabolism does not involve the cytochrome $\mathrm{P} 450$ system (CYP). Thus, in order to understand the potential of the new oral anticoagulants to generate drug interaction, we need to understand the transport of P-glycoprotein (P-gp). $\mathrm{P}$-gp functions as an efflux pump to prevent the absorption or increase the renal secretion of certain drugs known as p-GP substrates. Inhibitors of P-gp will increase the serum concentrations of P-gp substrates, and inducers of P-gp will reduce the serum concentrations of P-gp. Both dabigatran and rivaroxaban appear to be P-gp substrates. A review about dabigatran conducted by the European Medicines Agency states that amiodarone, an inhibitor of P-gp, increases the AUC of dabigatran by $60 \%$ and the Cmax by $50 \%$, and also recommends that the dose of dabigatran be reduced to $150 \mathrm{mg}$ daily in patients taking it concurrently with amiodarone. It further states that care must be taken with strong p-GP inhibitors like verapamil, clarithomycin and others, while quinidine is contraindicated. Potent $\mathrm{p}-\mathrm{GP}$ inducers such as rifampicin or St John's wort (Hypericum perforatum) may reduce the systemic exposure of dabigatran. Caution is advised when co-administering these medicinal products. There appears to be significant interplay between CYP3A4 and p-GP, such that they appear to act synergistically to limit oral drug bioavailability. Thus, drugs that are substrates for both CYP3A4 and p-GP often have low bioavailability. Moreover, drug interactions involving both CYP3A4 and $\mathrm{p}-\mathrm{GP}$ inhibition or induction may be more significant than those affecting one or the other system alone. Of the four drugs known to inhibit rivaroxaban metabolism by inhibiting CYP3A4, ketoconazole and ritonavir do so to the greatest extent. Those agents are strong inhibitors of both CYP3A4 and p-GP. Comparatively, erythromycin is a moderate inhibitor of both CYP3A4 and p-GP, while clarithromycin is a strong CYP3A4 and a moderate p-GP inhibitor (Wittkowsky, 2010).

\section{Hemorrhage complications}

No differences were found between dabigatran and enoxaparin, and between rivaroxaban and enoxaparin, in any category of bleeding, despite some inconsistencies among trials. The management of bleeding complications associated with traditional anticoagulants, including warfarin, UFH and LMWHs, is aided by the availability

TABLE I - Comparative pharmacokinetic properties of dabigatran and rivaroxaban

\begin{tabular}{ccc}
\hline Pharmacokinetic parameter/Drug & Dabigatran & Rivaroxaban \\
\hline Molecular weight & $628 \mathrm{Da}$ & $436 \mathrm{Da}$ \\
Protein binding & $35 \%$ & $>90 \%$ \\
$\mathrm{Vd}$ & $60-70 \mathrm{~L}$ & $50 \mathrm{~L}$ \\
Dialyzable & Yes & Not expected \\
Tmax & $1,25-3 \mathrm{~h}$ & $2,4 \mathrm{~h}$ \\
$\mathrm{~T} 1 / 2$ & $12-14 \mathrm{~h}$ & $5-9 \mathrm{~h}$ \\
Activation & Prodrug dabigatran etexilate converted to & None \\
& active drug dabigatran via hydrolysis & $36 \%$ \\
Renal excretion of unchanged drug & $80 \%$ & Oxidation (via CYP3A4 [18\%] and \\
Metabolism & Conjugation & CYP2J2 [14\%] and hydrolysis) \\
\hline
\end{tabular}

Vd: Volume of distribution; Tmax: Time to maximum serum concentration; $\mathrm{T}^{1 / 2}{ }_{2}$ : time of elimination half-life. (Wittkowsky, 2010). 
of antidotes (vitamin $\mathrm{K}$, protamine), laboratory tests to assess the level of anticoagulation (INR, aPTT, antiXa activity), and clinical guidelines. Comparatively, there is little information available at present to guide management of bleeding complications associated with the new oral anticoagulants nowadays. No specific antidotes are available and there is little information regarding the potential role of blood products, antifibrinolytic agents (aminocaproic acid, tranexamic acid) or prohemostatic agents, like recombinant factor VIIa, to manage bleeding associated with dabigatran or rivaroxaban. Methods to accelerate removal of these drugs from plasma using dialysis, hemoperfusion or plasmapheresis have yet to be investigated (Wittkowsky, 2010; Garcia, Libby, Crowther, 2010).

Evidence-based strategies for reversal of bleeding associated with new anticoagulants are not available. Although it may be necessary to intercede, it will be infrequently, because all drugs have short half-life times. To many clinicians, the lack of an antidote or an evidence-based strategy to manage bleeding may be a disadvantage. Principles for management of bleeding related to anticoagulants (with specific focus on new agents) have been described, at least in an in vitro study, where a potential strategy for further investigation was identified (Wittkowsky, 2010; Garcia, Libby, Crowther, 2010).

\section{Innovative drugs in development}

In addition to new therapeutic options present here, other anticoagulant drugs are still in development, or undergoing clinical trials. Although some of them have not been developed for use in VTE, DVT or PE, their applications in these pathologies should be evaluated.

In the class of VKA, tecarfarin appears as an important option for treatment of patients with atrial fibrillation, because it is less susceptible to drug interactions due to its metabolism by carboxylesterases, instead of warfarin, whose biotransformation is mediated by enzymes of $\mathrm{P} 450$ cytochrome system. This metabolic selectivity is possible due to the fact that tecarfarin is a single enantiomer. A phase II clinical assay proposed $15,6 \mathrm{mg}$ as mean daily dose, which was well tolerated by individuals participating of the trial (Ellis et al., 2010).

Recombinant human antithrombin (RHA) is a particularly important drug for patients who have hereditary deficiency of antithrombin production, and consequently need this medication to avoid thromboembolic events. RHA is administered together with heparinoids, and increases anticoagulant activity from 300 to 1,000 times. Its production is made through the use of transgenic goats, in which the human antithrombin gene is fused with regula- tory genes of mammary glands, producing about $2 \mathrm{~g}$ of RHA per L of milk (Cada, Levien, Baker, 2009).

In the universe of antidotes used to control bleeding, a new therapeutic approach is the prothrombin complex concentrate, containing factors II, VII, IX and X, in addition to anticoagulant proteins $\mathrm{C}$ and $\mathrm{S}$. This complex proved to be effective in reversing anticoagulation generated by administration of VKA (Pabinger et al., 2007).

\section{Recommendations}

Drug therapy in order to improve people's health does not end in the administration of a prescribed medication. Therapeutic follow-up based on clinical response and on the opinion of the patient regarding his clinical condition is decisive to evaluate the effectiveness of a drug. In this sense, pharmacovigilance plays an essential role, because a newly marketed drug still has not all necessary information, like drug interactions and adverse events. This condition comes from the limitations of clinical studies of phases I, II and III, which involve only selected patients, limited by both age and weight, with no other pathologies and not using other drugs.

In the first years of post-marketing drugs, it is extremely important to evaluate their efficacy and safety, because, during this period, several side effects and drug interactions of real importance but previously unknown are discovered. In addition, the description about medicines may change in the leaflets, and it may even be withdrawn from market, according to new data reported.

Responsible health professionals must always search information about drugs related to their work, in order that their professional performance can indeed improve the health and, consequently, the quality of life of population.

The total utility of all new anticoagulants will depend on many factors, including effectiveness compared with current agents, the cost, the benefit-risk profile, the reversibility of hemorrhagic events, convenience, and patient satisfaction. Generally, the results of safety and efficacy in clinical practice do not correspond to the controlled clinical trials of new anticoagulants, due to the several parameters that must be carefully observed;for example, unpredictable drug interactions, renal failure, obesity, a long half-life elimination of these agents, inability to monitor them practically and routinely, incapacity to quickly reverse their effects at the time of an emerging bleeding or invasive procedures, and other patient characteristics that may have a considerable influence on the results, limiting the use of these new substances. However, at moments such as prophylaxis after prolonged orthopedic surgery, these agents may replace LMWHs, and its 
oral administration and its cost can provide a persuasive argument for their use. Therefore, the entire team involved in drug therapy should be encouraged to carefully evaluate not only the dose and duration of therapy with these new anticoagulants, but also to monitor adverse effects and drug interactions in order to enjoy the conclusions about the real advantage of this therapy (Wittkowsky, 2010; Garcia, Libby, Crowther, 2010).

\section{CONCLUSION}

Currently, HPBM are the drugs of first choice for prophylactic treatment of DVT and VTE in orthopedic, multiple trauma and immobilized patients. These drugs have more effective and safety based on the widely studied clinical practice.

It is unquestionable that anticoagulant therapy has experienced many advances, particularly by integrating two new drugs with high level of importance. Undoubtedly, the new oral anticoagulants are likely to increase patient compliance compared with the injectable anticoagulant treatment. As discussed, clinical trials demonstrated noninferiority compared to enoxaparin in patients following orthopedic surgeries. In the future, with the expansion of the studies, rivaroxaban and dabigatran seem to emerge as potential drugs of choice for anticoagulation therapy.

\section{REFERENCES}

BREITENSTEIN, A.; TANNER, F.C.; LÜSCHER, T.F. Tissue factor and cardiovascular disease: Quo vadis? Circ. J., v.74, n.1, p.3-12, 2010.

CADA, D.J.; LEVIEN, T.L.; BAKER, D.E. Antithrombin (Recombinant). Hosp. Pharm. (Phila.), v.44, n.9, p.785790, 2009.

ELLIS, D.J.; USMAN, M.H.; MILNER, P.G.; CANAFAX, D.M.; EZEKOWITZ, M.D. The first evaluation of a novel vitamin K antagonist, Tecarfarin (ATI-5923), in patients with atrial fibrillation. Circulation, v.120, n.12, p.1029$1035,2009$.
GARCIA, D.; LIBBY, E.; CROWTHER, M.A. The new oral anticoagulants. Blood, v.115, n.1, p.15-20, 2010.

MAJERUS, P.W.; TOLLEFSEN, D.M. Coagulação sanguínea e anticoagulants, trombolíticos e fármacos antiplaquetários. In: BRUTON, L.L.; LAZO, J.S.; PARKER, K.L. (Eds.). Goodman \& Gilman: as bases farmacológicas da terapêutica. 10.ed. Rio de Janeiro: McGraw-Hill, 2006. p.1321-1339.

LASSEN, M.R., LAUX, V. Emergence of new oral antithrombotics: a critical appraisal of their clinical potential. Vasc. Health Risk Manag., v.4, n.6, p.1373-1386, 2008.

PABINGER, I.; BRENNER, B.; KALINA, U.; KNAUB, S.; NAGY, A.; OSTERMANN, H. Prothrombin complex concentrate (Beriplex $\left.{ }^{\circledR} \mathrm{P} / \mathrm{N}\right)$ for emergency anticoagulation reversal: a prospective multinational clinical trial. $J$. Thromb. Haemost., v.6, n.4, p.622-631, 2007.

RANG, H.P.; DALE, M.M.; RITTER, J.M.; MOORE, P.M. Pharmacology. 5.ed. Churchill Livingstone, Edinburgh: Elsevier, 2003. p.56-70.

SAMAMA, M.M.; GEROTZIAFAS, G.T. Newer anticoagulants in 2009. J. Thromb. Thrombolysis, v.29, n.1, p.92-104, 2010.

TURPIE, A.G. New oral anticoagulants in atrial fibrillation. Eur. Heart J. Suppl., v.29, n.2, p.155-165, 2007.

WALSH, G. Pharmaceutical biotechnology: concepts and applications. 1 ed. London: Willey, 2007. p.1-10.

WEITZ, J.I.; HIRSH, J.; SAMAMA, M.M. New antithrombotic drugs. Chest, v.134, n.2, p.473, 2008.

WITTKOWSKY, A.K. New oral anticoagulants: a practical guide for clinicians. J. Thromb. Thrombolysis, v.29, n.2, p.182-191, 2010.

Received for publication on $15^{\text {th }}$ February 2011 Accepted for publication on $15^{\text {th }}$ July 2011 